\title{
A POLÍTICA DE CONTROLO DE DOENÇAS TRANSMISSÍVEIS EM PORTUGAL
}

\author{
Denise Capela dos Santos
}

ORCID ID:0000-0002-5794-5203;

CIÊNCIA ID: 0B10-1C58-71EB,

dsantos@autonoma.pt

As infeções associadas aos cuidados de saúde e o aumento da resistência aos antimicrobianos representam um problema crescente em todo o mundo e Portugal não é exceção.

O país apresenta uma prevalência de infeções hospitalares elevada quando comparada com outros países da Europa, facto que tem uma implicação grande não só relativamente à segurança dos doentes como para a economia da saúde (Público, 2016)a ${ }^{\text {a }}$ Efetivamente, em caso de infeção hospitalar, o prazo médio de internamento aumenta de cerca de sete dias para mais de 36 dias, em hospitais públicos (Público, 2016)a ${ }^{\text {a }}$ havendo aqui um potencial enorme de redução da despesa do hospital associada ao combate às infeções nosocomiais.

Por outro lado, Portugal é, no contexto europeu, um país com elevada taxa de prescrição e de consumo de antibióticos, apesar de haver registo de uma melhoria deste indicador nos últimos anos (Loureiro et al., 2016).

O estudo do Controlo da Infeção e Resistência aos Antimicrobianos em Números, 2015, vem fundamentar que houve, em Portugal, em 2014, uma redução da incidência da pneumonia associada à intubação traqueal nas Unidades de Cuidados Intensivos, da bacteriemia associada a cateter venoso central e da infeção associada a cirurgia do cólon e reto. No entanto, verificouse um ligeiro aumento da incidência na infeção relacionada com a cirurgia das vias biliares (DGS, 2016) ${ }^{\circ}$, sugerindo que há ainda um longo caminho a percorrer para alcançarmos os valores de infeção pretendidos relativamente à prestação de cuidados. É por isso urgente investir em práticas de prevenção das infeções mais frequentes, principalmente nos hospitais.

O mesmo estudo registou evoluções positivas no consumo de antimicrobianos. O consumo de quinolonas desceu, entre 2011 e 2014, 27\% no ambulatório e mais de $8 \%$ a nível hospitalar; e o consumo de carbapenemos (antibióticos associados à seleção de bactérias multirresistentes) diminuiu 5\% entre 2013 e 2014.

No mesmo período, ainda de acordo com o Controlo da Infeção e Resistência aos Antimicrobianos em Números, 2015, verificou-se redução das taxas de resistência em alguns microrganismos multirresistentes, como Staphylococcus aureus, Enterococcus ou Acinetobacter, embora estas taxas se mantenham ainda elevadas. 
Working Paper

Departamento de Ciências Económicas e Empresariais

Universidade Autónoma de Lisboa

Preocupante é o caso da resistência elevada aos antibióticos da classe das quinolonas, pela Escherichia coli, bactéria responsável pela maior parte das infeções urinárias não complicadas, embora a principal ameaça seja proveniente da Klebsiella pneumoniae, resistente aos antibióticos da classe dos carbapenemos (DGS, 2016) .

Para dar resposta a esta situação, no Despacho n. ${ }^{\circ}$ 6401/2016 de 16 de Maio, o Governo incluiu o Programa de Prevenção e Controlo de Infeções e de Resistência aos Antimicrobianos (PPCIRA) no âmbito dos programas de saúde prioritários (DGS, 2016)a.

Este programa, criado pelo Despacho n. $.2902 / 2013$, de 22 de Fevereiro, tem como "objetivo geral a redução da taxa de infeções associadas aos cuidados de saúde, hospitalares e da comunidade, assim como a taxa de microrganismos com resistência aos antimicrobianos e a vigilância contínua da infeção hospitalar, do consumo de antibióticos e da incidência de microrganismos multirresistentes" (DGS, 2016) .

Para o sucesso deste programa, considera-se determinante o alerta para a melhoria dos níveis de literacia da população, para que o cidadão seja uma parceiro ativo na divulgação de boas práticas e na redução de comportamentos propícios ao contágio por doenças transmissíveis (DGS, 2016) ${ }^{\circ}$.

Outros programas definidos como prioritários, associados ao controlo de doenças transmissíveis são o de Infeção VIH/Sida e Tuberculose, e o de Hepatites Virais (DGS, 2016)a, indicando que estas são as patologias de cariz infeciosa a que o Governo pretende dar prioridade. De notar que, enquanto o Programa $\mathrm{VIH} / \mathrm{Sida}$ e Tuberculose já tinha sido criado anteriormente, o Programa de Hepatite Viral foi criado este ano. O novo programa pretende incentivar a prevenção da hepatite e desenvolver estratégias de controlo para esta doença, melhorar práticas de gestão da hepatite $C$ (nomeadamente em reclusos), e promover a monitorização e a vigilância da hepatite (Augusto e Fronteira, 2016).

Vejamos que dados se encontraram sobre as medidas implementadas pelo Ministério da Saúde, associadas à prevenção e ao controlo de infeções, assim como à prevenção e controlo de resistência aos antimicrobianos ou antibióticos.

\section{MEDIDAS IMPLEMENTADAS PARA PREVENÇÃO DE INFEÇÕES}

As boas práticas associadas à prevenção de infeções, de forma generalizada, têm a ver principalmente com uma boa educação sobre fontes, formas de contágio e estratégias para combate ou proteção contra doenças transmissíveis. 
Working Paper

Departamento de Ciências Económicas e Empresariais

Universidade Autónoma de Lisboa

Uma outra forma de prevenir infeções, embora de forma seletiva, é a vacinação. Apesar de ser um processo caro, tem a vantagem de conferir imunidade à população, permitindo evitar os custos associados a doenças específicas, que são em regra bastante superiores ao da vacinação respetiva. Infelizmente, não há vacinas para todos os agentes patogénicos causadores de elevada morbilidade ou mortalidade. No entanto, o Plano Nacional de Vacinação deve possibilitar a imunização da população (ou de grupos de risco, atendendo à verba disponível) contra as ameaças atuais de microorganismos com maior patogenicidade e prevalência no território.

O atual governo optou por alterar o Programa Nacional de Vacinação (PNV). Assim, em Janeiro de 2017:

1) a vacina contra o vírus do papiloma humano (HPV) passa a ser administrada às raparigas a partir dos 10 anos;

2) passarão a ser apenas com a BCG as crianças que pertencem a grupos de risco para a tuberculose;

3) a vacina contra o tétano passa a ser administrada aos 10,25, 45 e 65 anos de idade, e após os 65 anos, a vacina volta a ser administrada de $10 \mathrm{em} 10$ anos; e

4) início da vacinação das grávidas contra a tosse convulsa para a proteção dos recémnascidos (DGS, 2016) $)^{\mathrm{m}}$.

As referidas alterações pressupõem assim um aumento do investimento na proteção do cancro do colo do útero causado pelo HPV, na proteção do tétano e contra a tosse convulsa. Há, no entanto, um desinvestimento na prevenção da tuberculose. As vacinas BCG, distribuídas pelas Administrações Regionais de Saúde e pelas Regiões Autónomas dos Açores e da Madeira, a partir de Fevereiro deste ano, passam a destinar-se apenas a crianças com idade até aos 6 anos (5 anos e 364 dias), pertencentes a grupos de risco (identificadas e referenciadas a nível local pelas Unidades Saúde Pública de ACES/ ULA). Considera-se referentes a um grupo de risco as crianças provenientes de países com elevada incidência de tuberculose ou que tenham viagem planeada para esses países, que coabitem, ou convivam, com portadores de infeção VIH/SIDA e que apresentem dependência de álcool ou de drogas (DGS, 2016)d.

De acordo com o Diretor Geral da Saúde, a taxa de incidência de tuberculose em Portugal, em 2015, era de 19,2 casos diagnosticados e comunicados à Direção-Geral da Saúde (DGS) por cem mil habitantes, apesar das estimativas da OMS, assinalarem existir em Portugal a maior taxa de incidência de tuberculose da Europa Ocidental, com 23 casos por cem mil habitantes (Expresso, 2016). Será que há casos de tuberculose que não estão a ser devidamente diagnosticados ou comunicados à DGS? Sugere-se uma avaliação da discrepância de números entre estas fontes, que poderão levar a uma necessidade de reposição da vacinação universal pela BCG, considerando ainda os casos de tuberculose multirresistente que se têm registado em Portugal e no mundo, de muito elevado risco para a saúde pública. 
Ainda a propósito da vacinação, embora não pertença ao Plano Nacional de Vacinação, a Direção Geral de Saúde (DGS) coordenou a Campanha de Vacinação Contra a Gripe, em parceria com a Administração Central do Sistema de Saúde, com o Instituto Nacional de Saúde Doutor Ricardo Jorge, com as Administrações Regionais de Saúde e com os Serviços Regionais de Saúde das Regiões Autónomas dos Açores e da Madeira (DGS, 2016) ${ }^{c}$. Esta campanha inclui divulgação de informação sobre a vacina e a patologia associada, decisão sobre o grupo-alvo (de maior risco) considerando o montante disponível para a compra de vacinas e coordenação entre o pedido das vacinas à indústria, a receção das vacinas solicitadas pelos ACES/ ULS e a administração das vacinas à população.

Para tal, a DGS recomendou que todos os cidadãos com mais de 60 anos, doentes crónicos e imunodeprimidos, grávidas e profissionais de saúde, sejam vacinados contra a gripe (DGS, 2016) ${ }^{\text {e }}$ Contudo, nas Unidades de Saúde Familiares, a vacina manter-se-á gratuita apenas para os cidadãos com idade igual ou superior a 65 anos, para residentes em instituições ou na Rede de Cuidados Continuados Integrados, para doentes em diálise crónica e imunodeprimidos, grávidas e profissionais de saúde, sendo este ano alargada a doentes recetores de transplante e a aguardar transplante, a utentes a realizar tratamentos de quimioterapia, cidadãos com trissomia 21, doentes com fibrose quística, e pacientes com doença neuromuscular com comprometimento da função respiratória e com défice de alfa-1 antitripsina (DGS, 2016) $)^{\mathrm{e}, \mathrm{g}}$. Para dar resposta aos grupos populacionais citados, o Estado terá disponíveis, a partir de Outubro, cerca de 1,2 milhões de doses de vacinas de distribuição gratuita (DGS, 2016) ${ }^{\mathrm{c}}$.

No entanto, os cidadãos não abrangidos pela campanha gratuita poderão adquirir a vacina contra a gripe nas farmácias, com receita médica e beneficiar de uma comparticipação de 37\%. Nestes casos, a vacina custará cerca de 3,8 euros (DGS, 2016) .

Relativamente à educação dos cidadãos e atualização da informação a profissionais de saúde sobre fontes, formas de contágio e estratégias para combate ou proteção contra doenças transmissíveis, a DGS participou nas seguintes ações:

1. Divulgação do $13^{\circ}$ Encontro da Sociedade de Infeciologia Pediátrica, organizado pela Sociedade de Infeciologia Pediátrica, onde se discutirão temas como a epidemiologia e a efetividade da vacinação da tosse convulsa e doença invasiva pneumocócica (DGS, 2016) $)^{\mathrm{h}}$.

2. Lançamento de uma Campanha de prevenção dedicada à informação necessária para evitar riscos de surtos epidemiológicos associados a toxinfeções alimentares, no seguimento do Plano Verão \& Saúde. A adoção de medidas gerais de prevenção é importante, considerando que um alimento contaminado é muitas vezes difícil de ser identificado apenas pela sua cor, aspeto, cheiro ou sabor (DGS, 2016) .

3. Realização de um protocolo de colaboração com a Sociedade Portuguesa de Virologia para concretização de workshops com profissionais de várias regiões do país, com vista à "atualização dos principais tópicos relacionados com as infeções respiratórias, 
epidemiologia, etiologia, apresentações clínicas nos diferentes hospedeiros, diagnóstico, terapêutica e prevenção" (DGS, 2016) .

4. Deu orientações no sentido das Administração dos Hospitais promoverem a divulgação de informações aos doentes, aos visitantes e ao pessoal sobre riscos de aquisição e transmissão cruzada de infeções e a forma de os reduzir (DGS, 2016)'.

Ainda neste âmbito, o Instituto Ricardo Jorge e a Universidade Nova de Lisboa promoveram um workshop gratuito sobre "Doenças Transmitidas por Vetores", destinado a profissionais de saúde, investigadores e alunos da Universidade Nova de Lisboa, propiciando o desenvolvimento e promoção do conhecimento, assim como a colaboração em investigação na área das doenças transmitidas por vetores (DGS, 2016)'.

Há ainda a realçar o papel desempenhado pela Associação Portuguesa de Infeção Hospitalar (APIH), em colaboração com as ARS nacionais, no sentido da ampla divulgação das políticas associadas ao Programa de Prevenção e Controlo de Infeções e de Resistência aos Antimicrobianos (PPCIRA), através da realização de seminários de Prevenção e Controlo da Infeção - ERPI/Lares/UCCI, no território (APIH, 2016).

\section{MEDIDAS IMPLEMENTADAS PARA CONTROLO DE INFEÇÕES}

Para controlo de infeções, é importante alertar os profissionais de saúde dos hospitais, unidades de saúde familiar, unidades de cuidados continuados integrados, INEM, segurança dos aeroportos, escolas, etc. para a necessidade da rapidez do diagnóstico, notificação e cumprimento da estratégia de ação previamente planeada e articulada com as Unidades de Saúde Pública dos ACES/ ULS, com os departamentos de Saúde Pública das ARS e com o Centro Nacional de Saúde Pública do Instituto Doutor Ricardo Jorge, sempre que há contacto com um surto epidemiológico. É ainda preciso que haja uma boa rede de monitorização e de vigilância epidemiológica que permita tomar decisões com dados reais.

Para controlo de infeções foi publicado o Despacho n.11035-A/2016, que cria o Centro de Emergências em Saúde Pública na Direção-Geral da Saúde, para reforçar os sistemas de deteção precoce das ameaças sanitárias transfronteiriças, antecipando-as, bem como incrementar a capacidade de monitorização de indicadores e sinais de alerta, promover a comunicação em matéria de resposta e intensificar a respetiva capacidade de coordenação (DGS, 2016) $)^{\dagger}$. Apesar de ser importante a criação de um Centro de Emergência, considera-se este Centro deveria estar sobre a tutela do Instituto Doutor Ricardo Jorge, onde estão os especialistas nesta matéria, para que não haja duplicação de recursos nem sobreposição de 
Working Paper

Departamento de Ciências Económicas e Empresariais

Universidade Autónoma de Lisboa

funções. Considera-se sim fundamental estreitar a relação e partilha de informação online, em tempo real, entre as organizações.

Esta partilha de informação online foi bem sucedida, este ano, relativamente ao controlo da evolução dos dados epidemiológicos sobre o número de casos importados de Zika, em termos cumulativos, comunicados periodicamente à população num microsite sobre o Zika, disponibilizado pela DGS, para informação da população sobre o desenvolvimento da epidemia (DGS, 2016)j.

Da mesma forma, para maior controlo sobre as Infeções Hospitalares, site da Direção-Geral da Saúde e o Portal do Serviço Nacional de Saúde incluem informações e respetivos indicadores sobre as taxas de infeção em cada unidade hospitalar (DGS, 2016)'.

Atendendo à constatada necessidade de intervenção relativamente a este tipo de infeções, a Fundação Calouste Gulbenkian selecionou 12 unidades de saúde para o projecto STOP Infecção Hospitalar, feito em parceria com o Ministério da Saúde e o Institute for Healthcare Improvement, dos Estados Unidos. Nesta fase, integram o projeto as unidades de cuidados intensivos, as enfermarias de medicina e cirúrgicas dos centros hospitalares de Lisboa Central, Alto Ave, Barreiro-Montijo, São João, Cova da Beira, Lisboa Norte, Instituto Português de Oncologia do Porto, unidades locais de saúde de Matosinhos, do Nordeste e do Baixo Alentejo, Hospital de Braga e Serviço de Saúde da Região Autónoma da Madeira (Hospital Nélio Mendonça). O programa irá reforçar as medidas básicas de controlo da infecção - como a lavagem das mãos e o uso correto de luvas - e um programa que ajude os médicos a prescreverem melhor os antibióticos (Público, 2016)a.

É, no entanto, relevante estender estas medidas a todo o país logo que possível. Note-se que, após denúncia de que o hospital Garcia de Orta não isola doentes com bactérias multirresistentes, a DGS está a proceder a uma avaliação in loco do cumprimento dos procedimentos de prevenção e controlo de infeção, que poderão ter sido, neste caso, negligenciados. De acordo com a fonte, os doentes infetados com bactérias multirresistentes como a E-coli e a Klebsiela "estão misturados nas mesmas salas com os doentes que não estão infetados e inclusivamente com pacientes que foram submetidos a cirurgias" (Sábado, 2016), situação que, a verificar-se, é inadmissível nos dias de hoje.

Ainda no combate à propagação de infeções e contenção de surtos epidemiológicos, foi publicada a Portaria n 22/2016 de 10 de Fevereiro, que torna obrigatória a notificação de todos os laboratórios, a partir de 1 de Janeiro de 2017, dos casos de Doenças de Declaração Obrigatória através do SINAVE (Sistema Nacional de Vigilância Epidemiológica). Os Laboratórios passarão assim a integrar a rede de vigilância em saúde pública, destinada a identificar precocemente casos e surtos de doenças transmissíveis, suscetíveis de constituir uma 
Working Paper

Departamento de Ciências Económicas e Empresariais

Universidade Autónoma de Lisboa

emergência em Saúde Pública (DGS, 2016) ${ }^{n}$, medida de importante impacto na celeridade da resposta a eventual surto.

Por último, importa referir que a Direção-Geral de Saúde, em articulação com o Instituto Ricardo Jorge, elaborou um Plano estratégico de Prevenção e Controlo de Doenças Transmitidas por Mosquitos que define o conjunto de mecanismos que garantem a vigilância entomológica, a deteção precoce das populações de mosquitos invasores e na coordenação de resposta intersectorial (INSA, 2016), outra medida a salientar para o sucesso da vigilância epidemiológica.

\section{MEDIDAS IMPLEMENTADAS PARA REDUÇÃO DA RESISTÊNCIA AOS ANTIBIÓTICOS}

Os antibióticos ou antimicrobianos são medicamentos que matam ou que inibem o crescimento de bactérias, ajudando a curar infeções. As bactérias apresentam resistência aos antibióticos quando determinados antibióticos específicos perdem a sua capacidade de as matar ou de impedir o seu desenvolvimento. As bactérias resistentes são mais perigosas, sobrevivem na presença do antibiótico e continuam a multiplicar-se, causando uma doença mais prolongada ou a morte. $\mathrm{O}$ aparecimento e a propagação das bactérias resistentes é causado pela utilização inadequada de antibióticos, quando são usados no tratamento de infeções causadas por vírus, quando se encurta a duração do tratamento, quando de administra uma dose sub-terapêutica ou quando não se cumpre o intervalo de tempo correto de administração, por exemplo (ECDT, 2016).

Manter a eficácia dos antibióticos e reduzir a resistência das bactérias é assim uma responsabilidade de todos, tanto de médicos (que devem optar primeiramente por prescrever antibióticos de primeira linha, de pequeno espetro e apenas quando estritamente necessário, e educar os pacientes para o cumprimento integral do tratamento) como de pacientes (que devem respeitar sempre os conselhos dos médicos e realizar os tratamentos até ao fim, que não devem tomar "sobras" de antibióticos quando se sentem doentes nem deitar essas "sobras" no lixo doméstico, e que devem lavar as suas mãos e as dos seus filhos com alguma frequência) (ECDT, 2016). Todas as sobras de antibióticos ou de outros medicamentos fora de prazo, devem ser entregues nas farmácias das respetivas localidades e depositados nos caixotes Valormed.

Como contributo para a utilização racional dos antibióticos, em Fevereiro de 2016, o Ministério da Saúde, a Associação Portuguesa da Indústria Farmacêutica (Apifarma), a Associação Portuguesa dos Medicamentos Genéricos e Biossimilares (Apogen), a Associação de Grossistas de Produtos Químicos e Farmacêuticos (Groquifar), a Associação Nacional das Farmácias (ANF), a Associação das Farmácias de Portugal (AFP) e a Associação Portuguesa das Empresas dos Dispositivos Médicos (Apormed), assinaram um Compromisso para a Sustentabilidade e o Desenvolvimento do Serviço Nacional de Saúde. Este Compromisso "decorre do objetivo da 
Working Paper

Departamento de Ciências Económicas e Empresariais

Universidade Autónoma de Lisboa

promoção de uma política sustentável na área do medicamento e produtos de saúde, conciliadora do rigor orçamental com o acesso, a equidade, a utilização racional e segura dos medicamentos, a sua qualidade e possibilidade de introdução no mercado Português de terapias farmacológicas inovadoras" (Ministério da Saúde, 2016). É preciso, no entanto, avaliar, num futuro próximo, que resultados concretos se alcançaram através desta parceria.

Uma medida importante para os objetivos do país provém da direção do Programa de Prevenção e Controlo de Infeção e de Resistência aos Antimicrobianos, que "pretende que os hospitais e restantes unidades de saúde que contribuam para um uso mais racional de antibióticos ou que tenham menos infeções associadas à resistência a estes medicamentos passem a receber compensações financeiras no futuro" (Público, 2016) . Seria importante preceder à implementação desta medida quanto antes.

\section{CONCLUSÃO}

É determinante continuar a revitalizar o programa de controlo das doenças transmissíveis para enfrentar novas epidemias, a recrudescência de infeções conhecidas e a resistência múltipla aos antibióticos.

Para controlo das doenças transmissíveis, entende-se como prioritário avaliar periodicamente os procedimentos dos hospitais e de unidades de cuidados continuados relativamente às boas práticas no combate às infeções, assim como se considera importante aplicar coimas às organizações quando, depois de alertados para más condutas, se verificar que as más práticas se perpetuam, comportando um risco para a saúde pública dos Portugueses.

É ainda preciso atualizar e sensibilizar os profissionais de saúde face a esta temática, assim como educar os cidadãos para se protegerem de doenças contagiosas e para fazerem um uso racional e seguro de todos os medicamentos e nomeadamente dos antibióticos.

Salienta-se a enorme importância da colaboração de todas as pessoas e organizações públicas, privadas e do setor social, nas tarefas que venham a ser definidas para cada sistema de vigilância epidemiológica. Considera-se que este sistema deveria ser coordenado pelo instituto de maior referência nacional em epidemiologia e saúde pública, o Instituto Nacional Doutor Ricardo Jorge e descentralizado a nível regional (departamentos de saúde pública das ARS) e a nível local (unidades de saúde pública por ACES/ ULS), havendo uma responsabilização partilhada pelo alcance de melhores resultados relativamente às taxas de infeções e à utilização racional dos antibióticos, em todo o país.

\section{REFERÊNCIAS BIBLIOGRÁFICAS}


APIH (2016). $7^{0}$ Seminário "Prevenção e Controlo da Infeção - ERPI/UCCI. Recuperado a 5 de Novembro de 2016, de http://www.apih.pt/

Augusto, G. e Fronteira, I. (2016). Novos Programas de Saúde Prioritários. Online HIT, Chapter 5 - Section 5.1. Recuperado a 5 de Outubro de 2016, de http://www.hspm.org/countries/ portugal25062012/countrypage.aspx

DGS (2016) a Programas de Saúde Prioritários. Recuperado a 5 de Outubro de 2016, de https://www.dgs.pt/programas-de-saude-prioritarios.aspx

DGS (2016)b. Programa de Prevenção e Controlo de Infeções e de Resistência aos Antimicrobianos. Recuperado a 5 de Outubro de 2016, de https://www.dgs.pt/programa-deprevencao-e-controlo-de-infecoes-e-de-resistencia-aos-antimicrobianos.aspx

DGS $(2016)^{c}$. Vacinação contra a gripe 2016/2017. Recuperado a 5 de Outubro de 2016, de http://www.portugal.gov.pt/media/21549811/vacinacao-contra-a-gripe-2016-2017.pdf

DGS $(2016)^{d}$. Vacina da BCG começa a ser distribuída. Recuperado a 5 de Outubro de 2016, de http://www.portugal.gov.pt/pt/ministerios/ms/noticias/20160212-ms-bcg.aspx

DGS (2016) $)^{\mathrm{e}}$. Vacina contra a gripe recomendada a cidadãos com mais de 60 anos. Recuperado a 5 de Outubro de 2016, de http://www.portugal.gov.pt/pt/ministerios $/ \mathrm{ms} /$ noticias/20161003ms-vac-gripe.aspx

DGS (2016) $)^{\dagger}$. Criação do Centro de Emergências em Saúde Pública. Recuperado em 5 de Outubro de 2016, de https://www.dgs.pt/em-destaque/criacao-do-centro-de-emergencias-emsaude-publica.aspx

DGS $(2016)^{9}$. Mais doentes com vacina contra a gripe. Recuperado a 5 de Outubro de 2016, de https://www.dgs.pt/em-destaque/mais-doentes-com-vacina-contra-a-gripe-gratuita.aspx

DGS $(2016)^{\mathrm{h}}$. $13^{\circ}$ Encontro da Sociedade de Infeciologia Pediátrica. Recuperado em 5 de Outubro de 2016, de https://www.dgs.pt/em-destaque/13-encontro-da-sociedade-deinfeciologia-pediatrica.aspx

DGS (2016)i. Workshop sobre Doenças Transmitidas por Vetores. Recuperado em 5 de Outubro de 2016, de https://www.dgs.pt/em-destaque/workshop-sobre-doencas-transmitidas-porvetores1.aspx

DGS (2016)j. Doença por vírus Zika. Recuperado em 5 de Outubro de 2016, de https://www.dgs.pt/em-destaque/doenca-por-virus-zika-atualizacao.aspx

DGS $(2016)^{k}$. Como evitar o risco de toxinfeções alimentares. Recuperado a 5 de Outubro de 2016, de https://www.dgs.pt/em-destaque/como-evitar-o-risco-de-toxinfecoes-alimentares .aspx

DGS (2016)' Comunicado da Direção Geral de Saúde: Infeções hospitalares. https://www.dgs.pt/a-direccao-geral-da-saude/comunicados-e-despachos-do-director-geral/ infecoes -hospitalares.aspx

DGS $(2016)^{\mathrm{m}}$. Novo Programa Nacional de Vacinação. Recuperado em 5 de Outubro de 2016, de https://www.dgs.pt/em-destaque/novo-programa-nacional-de-vacinacao2.aspx 
DGS (2016)n. Notificação laboratorial obrigatória de doenças transmissíveis. Recuperado a 5 de Outubro de 2016, de https://www.dgs.pt/em-destaque/notificacao-laboratorial-obrigatoria-dedoencas-transmissiveis.aspx

DGS $(2016)^{\circ}$. Portugal - Controlo da Infeção e Resistência aos Antimicrobianos em números 2015. Recuperado a 5 de Outubro de 2016, de https://www.dgs.pt/em-destaque/portugalcontrolo-da-infecao-e-resistencia-aos-antimicrobianos-em-numeros-2015.aspx

DGS (2016) . DGS assina protocolo de colaboração com Sociedade Portuguesa de Virologia. Recuperado a 5 de Outubro de 2016, de https://www.dgs.pt/em-destaque/dgs-assinaprotocolo-de-colaboracao-com-sociedade-portuguesa-de-virologia.aspx

ECDT (2016). Ficha Informativa para o Público em Geral. European Center for Disease Prevention and Control. Recuperado a 12 de Novembro de 2016, de http://ecdc.europa.eu/pt/eaad/antibiotics-get-informed/factsheets/pages/general-public.aspx

Expresso (2016). Francisco Jorge contesta números sobre tuberculose em Portugal. Recuperado a 12 de Novembro de 2016, de http://expresso.sapo.pt/sociedade/2016-10-13-FranciscoGeorge-contesta-numeros-sobre-tuberculose-em-Portugal

INSA (2016). Plano de Prevenção e Controlo de Doenças Transmitidas por Mosquitos. Recuperado a 10 de Novembro de 2016, de http://www.insa.pt/sites/INSA/Portugues /Comlnf/Noticias/Paginas/PPCDTMosquitos.aspx

Loureiro, R. et al. (2016). O uso de antibióticos e as resistências bacterianas: breves notas sobre a sua evolução Revista Portuguesa de Saúde Pública, Volume 34, Issue 1, Pages 77-84

Ministério da Saúde (2016). Farmácias e indústrias farmacêutica e de dispositivos médicos assinam compromisso de sustentabilidade com o governo. Recuperado a 5 de Outubro de 2016, de http://www.portugal.gov.pt/pt/ministerios/ms/noticias/20160225-mssustentabilidade.aspx

Público $(2016)^{a}$. Hospitais integram projecto para reduzir infecções hospitalares para metade. Recuperado a 10 de Novembro de 2016, de https://www.publico.pt/sociedade/ noticia/hospitais-aderem-a-projecto-para-reduzir-infeccoes-hospitalares-para-metade1690884

Público (2016) ${ }^{\text {b }}$. Hospitais com melhor uso de antibióticos vão ter compensação financeira. Recuperado a 10 de Novembro de 2016, de https://www.publico.pt/sociedade/noticia /hospitais-com-melhor-uso-de-antibioticos-vao-ter-compensacao-financeira-1714597

Sábado (2016). Bactérias. DGS faz inspecção ao Garcia de Orta. Recuperado a 10 de Novembro de 2016, de http://www.sabado.pt/portugal/detalhe/bacterias_dgs_faz_inspeccao_ao _garcia_de_orta.html 